\title{
Editorial
}

Nephrology

Published online: November 13, 2018

DOI: 10.1159/000494805

\section{Intradialytic Hypotension: Is Midodrine the Answer?}

\author{
Mary Hammes $^{\text {a }}$ George L. Bakris ${ }^{b}$ \\ ${ }^{a}$ Department of Medicine, Section of Nephrology, University of Chicago Medicine, Chicago, IL, USA; ${ }^{b}$ Department of \\ Medicine, Comprehensive Hypertension Center, University of Chicago Medicine, Chicago, IL, USA
}

Dialysis-associated hypotension is very common, occurring in over $20 \%$ of hemodialysis treatments, and contributes significantly to morbidity and mortality in the end-stage renal disease (ESRD) population [1]. Intradialytic hypotension (IDH) is defined as systolic blood pressure (BP) less than $100 \mathrm{~mm} \mathrm{Hg}$ or a greater than 20 $\mathrm{mm} \mathrm{Hg}$ drop in BP with clinical symptoms of dizziness, blurred vision, hoarseness, cramps, and or syncope. Acute hypotension can lead to end-organ hypoperfusion with resultant cardiac ischemia, arrhythmia, sudden death, and altered gastrointestinal and neurologic function. Altered neurologic function and changes in cerebral architecture are observed with repeated episodes of IDH.

The pathogenesis of IDH is poorly understood but summarized in a recent review [2]. Multiple factors are implicated as contributing to IDH pathophysiology and include the following: (a) rapid fluid removal with sudden reduction in cardiac refilling during the hemodialysis session, (b) pre-existing autonomic neuropathy, (c) acute change in serum osmolality, (d) blunted response to vasopressin, (e) impaired baroreceptor sensitivity, and (f) impaired sympathetic activity with increased sensitiv-

\section{KARGER}

(c) 2018 S. Karger AG, Basel

E-Mail karger@karger.com

www.karger.com/ajn ity to the Bezold-Jarisch reflex [2-4]. According to the United States Renal Data System, the majority of incident and prevalent hemodialysis patients over the age of 65 years have underlying diabetes, hypertension, asymptomatic heart failure, or related conditions contributing to diastolic dysfunction. All these also contribute directly or indirectly to the development of autonomic dysfunction.

There are several options to treat IDH including prolonging treatment time on dialysis, more frequent dialysis, adherence with the dialysis schedule, lower dialysis bath temperature, and use of the alpha agonist, midodrine $[2,5]$. Midodrine is a selective $\alpha-1$ adrenergic receptor agonist, which causes peripheral arterial vasoconstriction and increased venous return. It was first available for clinical use of dysautonomia and orthostatic hypotension in 1996. Given the common problem of IDH, it is now often prescribed to ESRD patients and given before dialysis to lessen the chance of an adverse event during the hemodialysis treatment.

In this issue of the journal, Brunelli et al. [6] performed a retrospective review of 1,046 patients prescribed midodrine and compared cardiovascular outcomes to 2,037 
matched controls. Patients were matched for dialysis vintage, pre-dialysis systolic BP, monthly nadir systolic BP, and percentage of treatments affected by IDH. Both groups were followed to determine clinical outcomes. Compared to control patients, midodrine use resulted in higher death rates, all-cause hospitalization, and cardiovascular hospitalizations. While the underlying reason for prescribing midodrine was not disclosed and medication adherence was not directly assessed, this was a compelling study given the large cohort studied. It raises the question of whether midodrine use genuinely improves cardiovascular outcomes of hemodialysis patients. This study provides good evidence that while midodrine enhances the likelihood of effective volume removal during hemodialysis, it does not reduce mortality or adverse long-term outcomes. While past studies report the safety and efficacy of acute midodrine use, this is the first study to evaluate midodrine use on long-term outcomes and mortality.

Previous studies evaluating midodrine use for IDH is plagued by minimal patient numbers. Prakash et al. [7] published a meta-analysis focused on the efficacy and safety of midodrine when used for IDH. In this analysis, all prospective and retrospective studies with at least 5 patients were included with results that supported midodrine as an effective and safe agent to ameliorate IDH. Cruz et al. [8] performed a randomized double-blind trial comparing midodrine with and without cool dialysis to treat hypotension. Their results showed that both cool dialysis and midodrine were effective strategies to ameliorate IDH but there was no additive benefit when used together. Thus, the current work by Brunelli et al. [6] extends our knowledge in this area by demonstrating an adverse long-term cardiovascular outcome with midodrine on mortality even though there is a benefit preventing hypotension to enable fluid removal.

Increased BP variability is well-known to be associated with higher stroke risk and increased cardiovascular events. Patients with autonomic dysfunction, by definition, have increased BP variability and higher cardiovascular risk. Selvarajah et al. [9] followed a cohort of incident ESRD patients for 6 years and noted that variability in systolic BP was a strong independent predictor of allcause mortality. The work of Brunelli et al. [6] suggests that trying to reduce such variability with an agent that is a short-acting alpha-agonist will increase BP variability significantly and, consequently, increase cardiovascular events.

It is instructive to determine the characteristics of ESRD patients susceptible to IDH. There are those with underlying diastolic dysfunction who are observed to have a $20-30 \mathrm{~mm} \mathrm{Hg}$ drop in BP after sizeable intradialytic volume removal. The second group has sustained asymptomatic hypotension with a systolic BP that rarely exceeds $100 \mathrm{~mm} \mathrm{Hg}$. The latter often have been on hemodialysis for many years without demonstrated cardiac dysfunction. Chronic hypotension, especially among those who have been on hemodialysis over many years may be secondary to inaccurate volume assessment from multiple access procedures, making an estimation of intravascular volume very challenging.

When establishing the diagnosis of IDH, a careful physical examination to determine volume status should be performed along with a thorough medication review and assessment of standing BPs. Due to increased arterial stiffness and multiple vascular accesses, these common measures often do not accurately reflect accurate volume removal optimal for an individual patient. If volume status is in question, an inferior vena cava diameter is easy to obtain and may serve as a useful indicator-need reference. Additionally, an accurate assessment of cardiac function is essential in understanding IDH, especially evaluating for pericardial disease as well.

While there is no single treatment that is best to reduce the magnitude of IDH limiting intradialytic weight gains, longer and more frequent dialysis sessions has been consistently effective. These are simple measures but often hard to achieve since both patient's and providers' usually reject these options. The maximum recommended ultrafiltration rate for hemodialysis is $13 \mathrm{~mL} / \mathrm{kg} / \mathrm{h}$.

Cooling of the dialysis to $34.4-35.6^{\circ} \mathrm{C}$ effectively decreases the occurrence of IDH through vasoconstriction and is an accepted modality reference. This approach is often fraught with passive resistance by patients because of chilling and discomfort. Many in-center dialysis units now provide heated recliners to provide maximum patient comfort to counteract this side effect; however, this partially counteracts the vasoconstriction that serves as the mechanism for improvement. Isolated ultrafiltration has been shown to prevent IDH as the fluid removed is isotonic to plasma resulting in a minimal change in plasma osmolality. If the above efforts to prevent IDH hypotension fail, and underlying cardiac pathology is excluded, other approaches such as assuming a Trendelenburg position should be tried. Midodrine is the last option that should be tried to allow for additional volume removal during hemodialysis. Given this latest data, however, mi- 
dodrine should only be used as a last resort in ESRD patients.

In summary, IDH is a common and significant problem that we need to address daily in the care of hemodialysis patients. While midodrine appears to be a safe and effective treatment for IDH in the short term, the most recent evidence suggests it may be associated with increased mortality and mortality in the long term. It is wiser, therefore, to select other options discussed earlier and leave midodrine as a last resort. A long-term, adequately powered prospective trial to determine short- and longterm outcomes and risk of midodrine use to treat IDH in ESRD patients is needed.

\section{Disclosure Statement}

The authors have no conflict of interests.

\section{References}

1 Shoji T, Tsubakihara Y, Fujii M, Imai E: Hemodialysis-associated hypotension as an independent risk factor for two-year mortality in hemodialysis patients. Kidney Int 2004;66: 1212-1220.

2 Reeves PB and Mc Causland FR: Mechanisms, clinical implications, and treatment of intradialytic hypotension. Clin J Am Soc Nephrol 2018;13:1297-1303.

3 Chesterton LJ, McIntyre CW: The assessment of baroreflex sensitivity in patients with chronic kidney disease: implications for vasomotor instability. Curr Opin Nephrol Hypertens 2005;14:586-591.
4 Lazarus JM, Hampers CL, Lowrie EG, Merrill JP: Baroreceptor activity in normotensive and hypertensive uremic patients. Circulation 1973;47:1015-1021.

5 Flythe JE: Ultrafiltration rate clinical performance measures: ready for primetime? Semin Dial 2015;29:425-434.

6 Brunelli SM, Cohen DE, Marlowe G, Van Wyck D: The impact of midodrine on outcomes in patients with intradialytic hypotension. Am J Nephrol 2018;48:381-388.
7 Prakash S, Garg AX, Heidenheim AP, House AA: Midodrine appears to be safe and effective for dialysis-induced hypotension: a systematic review. Nephrol Dial Transplant 2004;19:2553-2558.

8 Cruz DN, Mahnensmith RL, Brickel HM, PerazellaMA:Midodrineand cooldialysateareeffective therapies for symptomatic intradialytic hypotension. Am J Kidney Dis 1999;33:920-926.

9 Selvarajah V, Pasea L, Ojha S, Wilkinson IB, Tomlinson LA: Pre-dialysis systolic blood pressure-variability is independently associated with all-cause mortality in incident haemodialysis patients. PLoS One 2014;9:e86514. 